\title{
POLITICAS PUBLICAS Y MUJER JOVEN: ENTRE LA MADRE Y LA HIJA*
}

\author{
ASTRID OYARZÚN CHICUY**
}

\section{PRESENTACión}

«POLÍTICAS PÚBLICAS Y MUJER joven: entre la madre y la hija», es el nombre que he dado a esta ponencia, pensando que la construcción de una política pública para ellas se ha movido o está entre las concepciones que se abordan desde la perspectiva de la mujer adulta, a la que le estoy dando la connotación de madre y, aquellas que arrancan desde una sociología de la juventud y que estoy llamando la hija. En mi opinión, ninguna de las dos perspectiva ha logrado realizar una construcción del sujeto mujer joven o de la joven mujer; y a partir de ello levantar una política social y pública para más del cincuenta por ciento de la juventud chilena. Ésta será la dirección que tomará este texto, tratando de demostrar, más bien, la ausencia de la mujer joven en lo que llamamos hoy en día política social pública. Desde luego, y por razones obvias, mi análisis estará más centrado en la perspectiva joven que en la mujer.

\footnotetext{
* Ponencia presentada en el seminario «Mujer joven: revelando lo invisible», organizado por el SERNAM V Región y CIDPA, en Viña del Mar, septiembre del 2000.

**_Asistente Social, CIDPA Viña del Mar. E-mail: astrid@cidpa.cl.
} 


\section{PRODUCCIÓN DE CONOCIMIENTO}

Haciendo un recorrido y revisión de la producción de conocimiento en la últimas dos décadas, uno podría decir que todo ese conocimiento ha contribuido a poner en el debate público los temas de la mujer, eso es un hecho. El conocimiento producido ayudó a gestar el sentimiento de pertenencia a un género y a definir nuevos problemas o más bien a identificar los problemas de ellas, de las mujeres adultas: violencia doméstica, jefatura femenina, derechos reproductivos, desigualdades ante la ley, feminización de la pobreza, entre otros. Gracias a la investigación fue posible argumentar la singularidad de sus experiencias sociales, diferentes a la de los hombres, subrayar su contribución social y conceptualizar los problemas que ellas enfrentan en tanto mujeres. De su parte, la acción política de los distintos grupos sociales y la difusión del conocimiento producido, interpeló a las fuerzas políticas y sociales a reflexionar sobre el curso y significado del movimiento de mujeres para la construcción de la democracia, y a las autoridades públicas a atender las manifestaciones más extremas de la subordinación de las mujeres y de la exclusión de ellas en el escenario social, político, económico y cultural. ${ }^{1}$ En el caso de nuestro país, ello se ve cristalizado, entre otras cosas, en lo que hoy conocemos como el Servicio Nacional de la Mujer (SERNAM).

En Chile y América Latina en general, efectivamente las mujeres jóvenes han estado en los estudios producidos por las ciencias sociales. Sin embargo, su presencia no ha pasado de ser marginal debido al bajo número de estudios, ${ }^{2}$ por un lado, y por otro, al tipo de

1 Virginia Guzmán: «La equidad de género como tema de debate y de políticas públicas». En Eliana Largo (editora): Género en el Estado, estado del género, ISIS Internacional, Santiago, 1998, p. 57.

2 El Instituto Nacional de la Juventud entre los años 1996 y 1999, ha realizado sólo dos estudios relacionados con la mujer joven. El primero, en el año 1997, que tuvo por nombre «Caracterización de las jóvenes beneficiarias del Programa Chile Joven y su inserción laboral». El segundo, también del año 1997, «Caracterización diagnóstica de la realidad de las mujeres jóvenes en Chile». Otro tipo de estudios, han estado fuertemente relacionados con la temática de la sexualidad, especialmente el tema del embarazo en adolescentes. En el caso del SERNAM, entre los años 1997 y 1999, se verifica la publicación de cuatro trabajos: «Análisis de las conductas sexuales de los y las adolescentes de la Región Metropolitana», «Apoyo a la gestión de políticas públicas. Evaluación y propuesta, embarazo en adolescentes», «Prevención del embarazo en adolescentes. Una mirada desde el Estado» y «Catastro de instituciones y/o programas para adolescentes». 
enfoque que se ha utilizado para su tratamiento: análisis a partir de las concepciones de mujer adulta — no reconociéndose su especificidado en las de juventud — analizándola como si fuera asexuada-, en las que privan los enfoques teóricos del universo masculino. Nos encontramos entonces, con un grupo humano que se ha caracterizado históricamente por su silencio, su invisibilidad y, en última instancia por su ausencia.

Por otro lado, habría que decir, que los primeros estudios que se realizan en la temática de la mujer, se desarrollan a partir del Año Internacional de la Mujer y del Decenio de la Mujer, es decir, durante los años 80. Posterior a eso, la continuidad de estudios asociados a la mujer joven se realizan a partir de la perspectiva de juventud. En nuestro país, ha sido una constante la combinación de ambos.

También habría que decir que según algunas estudiosas de esta materia, se pueden identificar cinco etapas por las que atravesaron los estudios sobre la mujer y que han pauteado el ciclo seguido por la investigación sobre mujeres jóvenes en América Latina y en Chile. ${ }^{3}$

i) La incorporación de mano de obra femenina en el mercado de trabajo. Es decir, estudios que analizaron las diversas formas de participación de la mujer en la fuerza de trabajo, en el campo y la ciudad. Estudios que presentaron sus resultados estableciendo cortes etáreos y, donde tal metodología constituyó, y constituye una forma de ir incorporando la situación de la mujer joven.

ii) Aquellos estudios teóricos inspirados en una sociología más bien de corte marxistas, que intentan realizar un análisis y tratamiento de la cuestión de la reproducción en los análisis sobre producción, procurando entender la relación de ambos términos.

iii) Estudio relacionados con las temáticas del trabajo y la familia. Aparece la preocupación por el impacto del cambio del estatus social de la mujer y su creciente incorporación en el mercado de trabajo. Por lo mismo, aparecen también estudios sobre salud de la mujer, en especial sobre el aborto, la contracepción, la planificación familiar, preocupaciones que surgen a propósito de los análisis centrados en el trabajo. Éste constituye un tipo que comienza a ser un aporte interesante en lo que luego alcanzará el nivel de políticas públicas para las mujeres, y también para los jóvenes.

iv) Los estudios que destacan el rol de la mujer en las estrategias de supervivencia o sobrevivencia, en la reproducción de la fuerza

3 Virginia Guzmán: Op. cit. 
de trabajo, en la jefatura de la familia. Son los análisis sobre lo cotidiano, los que contribuyen de manera especial a dar visibilidad a la mujer en la familia, en el presente y en el pasado. Este tipo de enfoque, de alguna manera, abandona el tipo de estudios asociados a la doble opresión que les toca vivir a las mujeres: como clase y como género.

v) Los estudios relacionados con el ámbito de las ciencias políticas, atribuyéndolo, fundamentalmente, a la creciente organización política de las mujeres y a la fuerte presencia de ellas en los movimientos sociales, tanto en Chile como en América Latina durante los años 80.

Este fue de alguna manera el camino para abordar el tema de la mujer joven. Sin duda su contribución fue importante, aunque no se logró dar un enfoque adecuado y por tanto también un enfoque para la acción con este tipo de sujeto. Estoy hablando fundamentalmente de toda la década de los 80 .

Un segundo momento de las investigaciones que se realizan en torno a esta temática, más centrados en la década de los 90 , se caracterizarán por abordar su comprensión desde una doble especificidad: de mujer y de joven. Los estudios de mujer joven pasaron a comprenderla como jóvenes mujeres insertas en el mundo de la producción, de la educación, de la reproducción, de la participación y en el mundo de lo cotidiano. Se adoptó con más coherencia el principio sociológico de que el/la joven es un actor específico. Y el principio metodológico obligatorio en los estudios de juventud: de que las conductas juveniles difícilmente pueden ser analizadas si no se toma como referencia las dimensiones simbólicas de la vida social, es decir, sus motivaciones asociadas a la cultura, lo imaginario, la comunicación, el discurso, etc.

En el caso chileno, tanto el análisis de la temática juvenil en general, como la de la joven mujer en particular, ha tenido también algunos problemas. En el caso de la perspectiva juvenil, la producción de estudios durante la década de los 90 es bastante abundante. Sólo el Departamento de Estudios y Evaluación del INJ, durante los años 1996 y 1999, ha encargado la realización de 39 estudios y durante este mismo período lleva realizado dos Encuestas Nacionales de Juventud, con una tercera en curso. No voy a nombrar aquí las investigaciones de carácter sectorial — trabajo, educación, salud, drogas y otros-que se han realizado a partir de otros servicios públicos ni tampoco lo realizado por el mundo académico y no gubernamental. El dato que 
doy sólo pretende indicar que el tema joven ha adquirido una significativa visibilidad e interés en el debate público y académico en los últimos años.

Todas estas iniciativas de investigación han tenido, a lo menos, un propósito significativo, conocer más a los o de los jóvenes. No ha habido una década en que los jóvenes hayan sido uno de los objeto de más «interés» de las distintas disciplinas del conocimiento. Ello se explica, por un lado, por lo nuevo del tema, efectivamente, los jóvenes comienzan a tener relevancia social desde la década de los 70 en adelante. Pero también, porque este tipo de población, durante buena parte de la década de los 80 y toda la década de los 90 se ha caracterizado por hacerle ruido al sistema social, porque no logra ajustarse a los requerimientos que de ella se esperan. Estamos hablando específicamente de la capacidad de los jóvenes de integrarse al sistema social.

Pero además, porque ha sido en esta década, en donde más ha existido la posibilidad de levantar una política social pública especialmente para este sector, a propósito de la reestructuración de la política misma. Esto ha tenido ciertamente sus ventajas y desventajas, después de casi diez años de intento, especialmente en la dimensión mujer joven o joven mujer.

La característica fundamental de la gran mayoría de los estudios realizados en juventud, es que se elaboran no sobre una construcción teórica, sino a partir de los problemas juveniles construidos por las instituciones: es decir, a partir de una delimitación institucional, no sólo de la población, sino también de su problemática. ${ }^{4}$ Este rasgo permanece y se agudiza durante toda la década de los 90, su mejor ejemplo es, sin duda, el tema de los consumo, en concreto al de drogas y alcohol asociado casi en exclusividad al mundo juvenil y sobre todo al mundo juvenil popular. ¿Qué tienen que ver las mujeres jóvenes en este tema?

Lamentablemente, esta característica será una de los aspectos más importantes que da cuenta de la imposibilidad de construir teóricamente el objeto del estudio que estamos llamando joven mujer. Sobre manera el estudio de la dimensión de la mujer joven, y más aún, pues en ellas, a diferencia de los jóvenes en general, la delimitación institucional es mucho más restringida, a tal punto que identificamos a la mujer joven o a la joven mujer, sólo a partir de temas o problemas

4 Enrique Martín Criado: Producir la juventud. Editorial ISTMO, Madrid, 1998, p. 42. 
asociados a su sexualidad, como si ese fuese el único campo de identificación. De ahí las dificultades en la construcción de la sujeto joven mujer.

\section{LA DIMENSIÓN MUJER EN LA PERSPECTIVA JUVENIL}

Qué tan presente está la dimensión mujer en las perspectiva juvenil. Sigue vigente la pregunta que se formulara Teresa Valdés a mediados de los 80: ¿existe la juventud en la mujer, y qué es ésta, si es que existe? Si bien el número de estudios ha aumentado y, en líneas generales, la preocupación sobre los temas de mujer joven se ha incrementado, todavía su conocimiento es incompleto, y las políticas implementadas siguen siendo escasa y ofreciendo respuestas parciales a sus necesidades, ello porque su construcción como sujeto ha sido asimilada primero a las mujeres y después mujer joven o a los jóvenes y luego por agregación mujer joven. Una definición que hasta hace muy poco y, aún con dificultad, no toma en cuenta la variedad de diferencias que el concepto delínea. Por ejemplo, pocas veces se hace distinción de género, las definiciones pretenden pasar por asexuadas, aunque hacen referencia centralmente a los varones jóvenes, asimilando luego la de las mujeres jóvenes. Veamos algunas dimensiones en el caso de la temática juvenil.

La edad es uno de los aspectos en los cuales uno puede observar que su construcción se pensó primero en los varones y luego fue asimilada para las mujeres. En general, se dice que el límite inferior en la juventud está en los 14 años y el superior en los 24 y hasta en los 29 años dependiendo también de las definiciones institucionales. El límite inferior de edad se ha fijado en referencia a la serie de transformaciones psicobiológicas producidas en la pubertad. Sin embargo, sabido es que el proceso de maduración es más precoz en la mujer que en el hombre. De ahí, el cuestionamiento que ha habido, por ejemplo, en torno al embarazo en adolescentes, en donde se fija el período de edad entre los 14 y 19 años de edad. Luego, según nuestra opinión la cota inferior de edad en la mujer joven podría o debería ser más baja, pero sin duda, esto no es un problema de número, sino que vuelve a ser éste un claro ejemplo de construcción de sujeto.

En el caso del límite superior de edad, su fijación corresponde a un teórico proceso que supone la finalización del período de maduración psicobiológica, la culminación de los estudios, el ingreso al

mercado laboral, la formación de la familia independiente y la plena 
asunción de las responsabilidades sociales. Es claro que el proceso de maduración de la mujer concluye antes de los 24 y mucho antes de los 29 años. La culminación de la preparación para los estudios ya se ha alcanzado a esa edad, y si se pudo acceder a estudios superiores éstos también ya se han realizado, baste ver las estadísticas. Cuando las mujeres pueden continuar sus estudios, son mejores alumnas y más responsables que los hombres. De modo, que «la edad juvenil» no estaría designando las mismas cosas para hombres y mujeres.

Otro aspecto en torno a este problema, dice relación con la moratoria social, un concepto que nuevamente ha estado más pensado en arreglo a la vivencia y experiencia masculina que a la femenina.

La moratoria hace referencia a aquel período de ensayo y error que le es permitido a los jóvenes, por el cual pueden destinar su tiempo a la formación, donde está permitido el idealismo, la aventura y los errores, y se extiende hasta que sean plenamente capaces, es decir, hasta la asunción total de sus responsabilidades sociales. Ello, incluso está legislado con diversos grados de precisión. La categoría de mayor de edad o ciudadano es a los 18 años, conducir también, casarse, entre otras. Sin embargo, la práctica social demuestra que a los hombres les están permitido muchas más cosas que a las mujeres jóvenes, sea en el plano del tiempo libre, como en el terreno sexual, la movilidad territorial, la asociación con sus pares, y que la sanción a posibles transgresiones es mucho más severa en el caso de las mujeres que en el de los hombres, llegándose en diversas circunstancias al cese precoz de la moratoria: piénsese en las exigencias que se plantean a la madre joven o a una madre soltera a quien se imponen rápidamente las exigencias de una adulta, no así en el caso de un padre joven o un padre soltero.

Otro aspecto es el referido a la construcción de una imagen pública. No existe una imagen pública de la mujer joven, y más precisamente no existe una creada por las propias jóvenes. Existe una baja definición de la imagen pública de la mujer joven, no así en el caso de la mujer o de los jóvenes en su referencia más masculina. Para reafirmar, es poco lo que sabemos de la mujer joven, pero eso escaso tampoco está trabajado a nivel de lo público.

Una de las razones es que la joven mujer, especialmente la de sectores populares, pasa buena parte de su vida en la reclusión del hogar, en donde es común la orientación de la mujer joven hacia el mundo privado y su baja participación en los escenarios sociales. Si el proceso de juventud se asocia a una etapa de tránsito, para una gran parte de las mujeres jóvenes, tal tránsito no es más que un paso entre 
mundos privados. De esta manera, pareciera ser que la imagen de la mujer joven pertenece más al mundo de la reclusión que al aspecto público, esto también es discutible en este tipo de análisis que estamos haciendo, donde la delimitación institucional juega un rol importante.

Algunos antecedentes de esta situación. Existe un Parlamento Juvenil, un símil en la expresión de la Cámara de Diputados. Los diputados adultos son 120, de ellos el $10.8 \%$ (13) son mujeres y el 89.2\% (107) hombres. En el caso de los senadores, de 49, sólo dos son mujeres (4.7\%). En cambio, de los 222 parlamentarios juveniles el $36 \%$ (80), son mujeres jóvenes, dirigentas jóvenes y el 64\% (142) son hombres. Es sabido también que quienes participan mayoritariamente en las organizaciones de Iglesia y estudiantiles, también son mujeres. Además son las mujeres jóvenes las que tienen una inclinación política definida y más opinión política que los hombres jóvenes.

Lo que quiero decir es que buena parte de la imagen de mujer joven que tenemos a tiempos actuales, se ha dado en gran medida por lo roles que tienen o vienen prefijados en su familia, los modelos que delínean los medios masivos de comunicación y por las características que le atribuyen otros sectores sociales: lo que estamos denominando la delimitación institucional. Esta es la situación que se ha ido tratando de cambiar, pero entrando ya al siglo XXI, la imagen de mujer joven que tenemos sigue conservando estas características, a pesar de ciertos antecedentes, hallazgos o investigaciones. Con estas imágenes y enfoques, se ha tratado de diseñar una política pública en los dos sentidos: por agregación al tema de la mujer adulta y/o por agregación al tema de política pública en juventud.

\section{LA POLÍTICA PÚBLICA SOBRE MUJER JOVEN}

La política pública sólo la abordaré desde la perspectiva de la política pública en juventud y lo que ha pasado en la década de los noventa en relación a la mujer joven.

Primero, decir algo de lo que entendemos por política social pública. La política social es la política pública orientada, desde el punto de vista administrativo, a la planificación y gestión de sectores sociales —educación, salud, vivienda, seguridad, social, deporte, recreación, etc.- y la atención de determinados grupos objetivos -niños, mujeres, jóvenes, discapacitados, ancianos, etc.- . Desde el punto de vista de la finalidad, ella persigue la reducción de las desigualdades sociales y la elevación del bienestar de la población, disminu- 
yendo los sectores que viven en situación de pobreza. ${ }^{5}$

Una de las razones de por qué se genera un espacio para la creación de una política social juvenil, viene de la mano con la necesidad de reorientar la política social en general, existente en el país hasta finales de los años 80. Es así que una de las grandes diferencias en el plano de las definiciones programáticas relativas a política social, entre el régimen militar y el régimen democrático, se encuentra en el cambio de prioridad estratégica referido a las áreas, enfoques, sectores y grupos sociales beneficiarios de los programas y recursos del gobierno. Constatando que el grupo-objetivo prioritario de la política social chilena ha sido históricamente el complejo madre-niño/a, ${ }^{6}$ el gobierno de la Concertación privilegiará, además, el que las inversiones sociales a temprana edad tengan posibilidades de madurar, ${ }^{7} \mathrm{y}$ por tanto, procurará proteger al estadio juvenil tanto como al de la niñez y la preñez.

Es en este contexto de cambios es que el sujeto juvenil, el de escaso recursos económicos, pasa a convertirse en un «grupo-objetivo», ${ }^{8}$ prioritario para el nuevo gobierno. Ya desde mediados de la administración de Patricio Aylwin es posible observar que la política social de juventud comienza a adquirir perfiles más definidos.

$5 \quad$ Flavio Cortés: «Fundamentos, característica e institucionalidad de la política social juvenil en Chile». Primer informe nacional de juventud. Instituto Nacional de la Juventud, Santiago, 1994, p.156.

6 Los programas referidos a la infancia y la preñez aparecen íntimamente asociados a la consolidación y desarrollo del Estado de Bienestar en Chile. $\mathrm{Al}$ respecto cabe consignar que el Programa de Salud Materna y Perinatal se asienta en una antigua preocupación del Ministerio de Salud por la protección médico y social de la maternidad. Así, en 1939, se creó el Consejo Superior de Protección a la Maternidad, a la Infancia y a la Adolescencia, lo cual derivó en la creación del Departamento Central de la Madre y el Niño del entonces Ministerio de Salubridad. J. Hurtado: «Programas estatales de salud dirigidos hacia las mujeres: imágenes de mujer y lógicas de acción». En T. Valdés y M. Bustos (editoras): Sexualidad y reproducción. Hacia la construcción de derechos. CORSAPS/FLACSO, Santiago, 1994, p. 7.

7 D. Raczynski et al.: «Políticas públicas de integración social. El caso chileno». Proyecto CLACSO sobre estrategias de gobernabilidad, Santiago, 1993, pp. 54-55, (sin publicar).

8 De acuerdo con Sergio Molina, «...el concepto de grupo-objetivo, comprende a los niños, los jóvenes, los adultos mayores, las mujeres, las personas con discapacidad y los pueblos indígenas. Ellos representan parte importante de la población que vive en condiciones de pobreza». Sergio Molina: Integración a desarrollo. MIDEPLAN, Santiago, 1994, p. 7. 
La mirada sobre los sectores juveniles, para los efectos del diseño de una política social, se centró en dos ejes teóricos-conceptuales. Por una parte, y desde un punto de vista sociológico, la noción de integración, proveniente de un enfoque más microsocial, y la concepción del daño o riesgo psicosocial. La primera se hacía tributaria de nociones como anomia social, mientras el concepto de daño aludía a una difusa condición social que afectaba a un desarrollo sano de la personalidad. ${ }^{9}$ La interrogante básica de por qué debería haber una política social juvenil, se respondió apelando al agudo cuadro de desinserción societal, traducido en desempleo y empobrecimiento de los sectores juveniles de bajos ingresos a finales de la década de los 80 .

Se planteó que el esfuerzo básico de una política social de juventud se debía dirigir a generar oportunidades de integración social de los jóvenes populares, en especial hacia el ámbito del empleo. ${ }^{10}$ Más aún, se planteó que una política social de juventud debe hacerse cargo de los dos desafíos centrales que atraviesan la fase juvenil. Por una parte, se trata de ofrecer oportunidades y brindar recursos para que los jóvenes puedan prepararse para enfrentar la vida adulta en el aprendizajes de roles que luego les tocará asumir. Como también debe orientarse a la satisfacción de las necesidades de conformación e integración de la personalidad, alcanzando el joven con ello, una clara y reconocible identidad social. Ambas dimensiones de una política de juventud se encuentran indisolublemente unidas y no puede subvalorarse ninguna de ellas. ${ }^{11}$ De igual modo, una política social juvenil debe privilegiar a los sectores sociales más pobres, permitiéndoles acceder a oportunidades que posibiliten un efecto compensatorio sobre la situación social de origen.

Comienzan así a aparecer en el debate público temas como el de la seguridad ciudadana, la apatía política, el rápido descenso en las tasas de inscripción electoral, la violencia en los estadios, el desempleo, el aumento en las tasas de drogadicción y alcoholismo, invadiendo la opinión pública a través de los medios de comunicación. Todos ellos son presentados como asuntos vinculados - directa o indirectamente- con los jóvenes y, en función de ello, a ser percibidos como trabas al proceso de transición a la democracia. En relación con estos fenómenos se plantea cada vez con más fuerza en el debate

$9 \quad$ Flavio Cortés: Op. cit., p. 159.

10 Flavio Cortés: Op. cit., p. 160.

11 Flavio Cortés: Op. cit., p. 161. 
público, temas como el de la pobreza, la marginalidad, la exclusión, la falta de oportunidades y de expectativas. Todos estos aspectos presentan un patrón común: la pobreza juvenil. Esta constatación, que deviene en la incorporación de nuevas definiciones estratégicas en relación con los jóvenes (la deuda social con los jóvenes), permite realizar a partir de la administración de Eduardo Frei Ruiz-Tagle, un replanteamiento general respecto del tipo de integración y participación que se desea.

Educación y trabajo aparecen como las dos grandes palancas del proceso de integración social de los jóvenes y, por ende, como la inversión a largo plazo de un país que accedía a la modernidad. Consecuentemente, la administración de Frei Ruiz-Tagle no introdujo modificaciones sustanciales a las anteriores orientaciones estratégicas; por el contrario, las acentuó. Ya en su mensaje presidencial del 21 de mayo de 1994, indicaba que la primera prioridad en su Programa de Superación de la Pobreza eran los niños y los jóvenes. ${ }^{12}$ En el caso de los jóvenes se trataba precisamente de darle un renovado énfasis a la capacitación laboral. Así, la integración por la vía del trabajo se mantiene como eje prioritario del tratamiento del mundo juvenil.

Preparar a los jóvenes para el mundo del trabajo cumplía una doble función en el proceso de integración social: una económica y otra social. No se descuida la producción de mano de obra necesaria para los nuevos requerimientos del mercado y se da paso a la integración de la juventud en el ámbito laboral. No obstante, plantear la integración social del joven excluido únicamente vía el ámbito laboral, parecía ser insuficiente y urgía mirar el problema integralmente.

Mirado en perspectiva el resultado de este primer ejercicio de readecuación no arrojó los resultados esperados. Los jóvenes no fueron preparados adecuadamente para el mundo del trabajo y mucho menos para ejercer sus derechos ciudadanos.

Problemas como los bajos resultados en el aprendizaje, la repitencia y deserción, la alta desigualdad social en función de las oportunidades educacionales, la baja eficiencia externa y el deterioro de la motivación docente, continúan manifestándose y su resolución no se vislumbra en el mediano plazo. ${ }^{13}$ Subyace la impresión que la inte-

12 «Mensaje Presidencial de su Excelencia el Presidente de la República, Eduardo Frei Ruiz-Tagle», Valparaíso, 21 de mayo de 1994. El Mercurio, Santiago, 22 de mayo de 1994.

13 M. J. Lemaitre: «El paso desde mejoramiento a reforma. Educación media en Chile, 1991-2001». En J. García-Huidobro (editor): La reforma educa- 
gración de los jóvenes, tanto hacia el mundo social como hacia el mundo educacional, no había sido lograda.

Se abre paso, a partir de ese momento, a un proceso de discusión que habría de desembocar en una radical redefinición de la política social de juventud, reconsiderando el espacio y la función del sistema educativo. Se asienta, especialmente en los documentos que fundamentaron el Programa MECE-MEDIA, la concepción de los jóvenes como co-constructores de la cultura escolar, a partir del reconocimiento de los saberes y quehaceres que identifican su entorno cultural. ${ }^{14}$ De esta manera, la Reforma Educativa pasa a convertirse en un requerimiento fundamental para el logro de la equidad social. ${ }^{15}$

Aparece además, un nuevo concepto en el espacio educacional: cultura juvenil y más concretamente, existe un llamado, una invitación a incorporar la cultura juvenil en los establecimientos educacionales como una manera de reforzar un aprendizaje participativo y ampliar la motivación estudiantil. ${ }^{16} \mathrm{Y}$ se realiza un balance de la incorporación de la cultura juvenil a la escolar: se señala la existencia de un desconocimiento generalizado de la cultura juvenil y la falta de una clara voluntad de incorporarla en el sistema educacional. La cultura escolar vigente - dice - tiende a considerar escasamente que los alumnos del nivel secundario constituyen un grupo etáreo singular, con sus códigos culturales y desafíos psicosociales específicos. Luego, realiza un examen crítico acerca del comportamiento juvenil popular en la enseñanza media: ${ }^{17}$ Veamos qué tanto tiene que ver esto con la joven mujer.

i) Mal uso del tiempo libre (fuera del liceo). El mal uso del tiempo libre fuera del liceo es considerado por el documento como un grave problema social, ya que se asocia no sólo con el ocio y la va-

cional chilena. Editorial Popular, Madrid, 1999, pp. 133-134.

14 Ministerio de Educación: Programa de mejoramiento de la calidad y equidad de la educación, 1995-2000. Fundamentos, estrategias y componentes, Volumen 1, Santiago, 1994, p. 86.

15 Al respecto la Comisión Nacional para la Modernización de la Educación, señalaba en 1995 que «entre el grupo de países con un desarrollo humano ‘alto' y 'medio', medido según el Índice de Desarrollo Humano de las Naciones Unidas, Chile es uno de los países que ostenta un menor gasto público en relación al PGB. Chile es uno de los pocos que invierte menos en educación en 1990 que en 1960». Comisión Nacional para la Modernización de la Educación, MINEDUC, Santiago, 1995, p. 53.

16 Ibid., pp. 13-15.

17 Ministerio de Educación: Op. cit., pp. 57-58. 
gancia, sino que también con la conducta tipificada del joven como riesgo social. Se relacionan con el «mal» uso del tiempo libre llamado aquí también «tiempo muerto»- los siguientes elementos: desarrollo de acciones rutinarias, pasivas y poco creadoras durante su tiempo libre, agrupamiento espontáneo en calles, formando los tipificados «grupos de esquina», altos índices de exposición a la TV, escasa o nula participación en organizaciones y grupos, o bien, de desarrollo sistemático de deportes, aumento de riesgos psicosociales asociados a las drogas, el alcohol y la violencia sin buscar el desarrollo personal. Búsqueda «transgresora» de salir de la monotonía, aburrimiento y falta de actividades.

ii) Mal uso del tiempo libre (dentro del liceo). El mal uso del tiempo libre dentro del liceo se asocia a una insuficiencia cuantitativa y cualitativa de actividades extraescolares. Los siguientes problemas se asocian a esta deficiencia del sistema escolar: impera una visión de que las actividades extraescolares son secundarias y marginales, la participación de los estudiantes en las actividades que existen es minoritaria, escasa incorporación de los intereses y gustos de los adolescentes y jóvenes, de lo anterior resulta una falta de identificación o sentido de pertenencia con el liceo, lo que promueve una desvinculación y desmotivación con la comunidad escolar general.

iii) Mal uso de la información entre el liceo y la realidad exterior. El mal uso de la información se asocia a un enclaustramiento del establecimiento y un aislamiento generalizado de los estudiantes con la realidad del mundo externo. Se exponen los elementos relacionados con esta forma de ver el problema de la información: poca fluidez en la relación con diversos actores y sectores externos al sistema escolar, escasez de vínculos con congéneres de otros establecimientos, padres y apoderados, marginación de las problemáticas sociales, creación de falsas expectativas, ya que predomina una orientación mayoritaria hacia la universidad, con una consecuente desvalorización de alternativas de trabajo existentes.

El potencial de riesgo social, la pérdida de identidad con el establecimiento y la creación de falsas expectativas en relación al futuro, constituyen todos ellos elementos derivados de las problemáticas juveniles vislumbradas por los diseñadores del programa señalado. Apuntan cada uno de ellos, hacia un mismo y claro diagnóstico de desconocimiento y no incorporación de la cultura juvenil a la cultura escolar. 
La aceptación de este hecho, refleja la apertura del debate acerca de la integración social juvenil popular hacia una visión que trasciende el ámbito meramente laboral. Con la ayuda de modelos teóricos y corrientes nuevas de las ciencias sociales, se abre el tema de la integración hacia la cotidianeidad de los jóvenes, su quehacer diario, sus intereses y visiones de futuro. De este modo, la aproximación oficial al problema hoy es otra y se aborda el tema de la integración en conjunto con el de la educación.

En este contexto general, del estado de la actual política social juvenil, es que tenemos o disponemos de distintos instrumentos a partir de los cuales se estaría generando un serie de programas destinados a verificar este proceso de integración social de los jóvenes.

\section{RELEVANCIA Y PRESENCIA DE LA MUJER JOVEN}

Como último aspecto, sería pertinente realizar algunas observaciones acerca de la relevancia y presencia de la mujer joven. Y lo primero que se podría indicar, es que no han sido pocos los esfuerzos realizados por ambos gobiernos de la Concertación en torno a la solución de los problemas de los jóvenes en general, si uno observa las cantidad de instrumentos o programas que se han ido preocupando de ello. Por un lado, la creación del Instituto Nacional de la Juventud, el Programa «Chile Joven», «el PROJOVEN», las «Casas y Oficinas de la Juventud», el FOSIS a través de los CDJ o PLDJ y sus derivaciones actuales, el SENAME a través de sus instrumentos de proyecto de prevención con subvención o por proyectos, el CONACE, el CONADI, y finalmente el Ministerio de Educación a través de su Componente Jóvenes. Sin embargo, a diez años de aplicación de la mayoría de estos instrumentos y de los recursos invertidos, la evaluación que se tiene es que ellos no han aportado a la integración social de los jóvenes, y mucho menos a la integración social de las mujeres jóvenes. Fundamentalmente por cuanto la concepción general de lo juvenil continúa atravesada por una percepción agregativa, que en el marco de la focalización, tiende a sectorizar programas y no a construir política de juventud. ${ }^{18}$

Esto en el caso de las mujeres, es aún más grave, pues sólo al inicio de la creación de la institucionalidad de juventud, se observó un

18 O. Dávila y C. Silva: «Políticas de juventud y su expresión en lo local». Última Década N¹1. Ediciones CIDPA, Viña del Mar, 1999, p. 142. 
ánimo o voluntad de dar un tratamiento especial a la mujer joven y su condición. De hecho, si uno revisa las iniciativas de la época a la fecha, nos encontraremos con las siguientes.

La creación del desaparecido PROJOVEN (1991), el Programa de Oportunidades para los Jóvenes, una iniciativa gubernamental, inédita en la tarea de contar con una política integral de desarrollo y ampliación de oportunidades para los jóvenes. Esta iniciativa estuvo formada por siete áreas - trabajo, educación, salud, protección y rehabilitación de menores y jóvenes, participación y derechos juveniles, recreación y uso creativo del tiempo libre y vivienda-, y en conjunto reunieron 44 programas dirigidos a la juventud, especialmente aquella de sectores populares. ${ }^{19}$ De todos los programas, sólo el área de salud con su programa de prevención del embarazo en adolescentes, dio cuenta de una dimensión particular de la mujer joven. Sin embargo, este programa ya en 1994 se encontraba discontinuado.

La creación del INJ en 1991. El que intentó a través de su Primer informe nacional de juventud, iniciar un proceso de discusión acerca de incluir la perspectiva de género en la planificación de las políticas sociales y de iniciar una relación nueva entre el Estado y la mujer joven, inexistente en la época y también en estos tiempos. En relación a los programas pilotos que pudo ejecutar en sus inicios, se observa que no existió ninguno destinado a la mujer joven, sólo fue una declaración de buenas intenciones que se ha venido repitiendo en el tiempo, pero que no ha significado el levantamiento de una política para ellas.

El Grupo de Trabajo Interministerial de Juventud (GTI), creado en 1992, con el objetivo de fortalecer una institucionalidad en materia de políticas de juventud y de realizar un diseño, ejecución y evaluación de los programas contenidos en el PROJOVEN. Esta instancia recién en 1999 da cuenta, en un documento de trabajo, de una caracterización y análisis de la política social gubernamental dirigida a los jóvenes. En ninguna de sus recomendaciones para el próximo período, aparece relevante la dimensión de género ni tampoco alguna mención especial en lo relativo a las problemáticas o necesidades de más de la mitad de la juventud chilena.

El Componente Jóvenes, del Programa MECE-MEDIA del Ministerio de Educación. Que nace en el marco de la reforma educativa

19 MIDEPLAN e INJ: El programa de oportunidades. PROJOVEN. MIDEPLAN e INJ, Santiago, 1993, p. 6. 
chilena y cuyo objetivo fundamental se platea como un proceso de integración de la cultura juvenil a la cultura escolar. Su subprograma de Actividades Curriculares de Libre Elección (ACLES) y que a esta altura de su recorrido sólo se han convertido en una oferta extraprogramática mejorada, no incidiendo para nada en la integración de la cultura juvenil, ni tampoco ha incidido en la temática mujer joven.

En definitiva, a mi juicio, considerando por un lado la producción del conocimiento de la juventud, por otro el tipo de enfoque que se está utilizando para hacer una lectura adecuada de la dimensión de género y el o los tipos de diseño de los instrumentos para diseñar un plan de acción en relación a ellas, queda claro que hasta ahora han prescindido de la mujer joven o la joven mujer: su ausencia en el marco de las políticas sociales y públicas es evidente. La propuesta en este ámbito tendrían que necesariamente pasar por hacer efectivamente una construcción de este sujeto y una adecuada expresión en la política social pública, ya sea al alero de la madre o de la hija.

VIÑA DEL MAR, MARZO DEL 2001 